The Journal of Nonlinear $\mathbf{S}_{\text {cience and Aplications }}$ http://www.tjnsa.com

\title{
MINIMAX INEQUALITY FOR A SPECIAL CLASS OF FUNCTIONALS AND ITS APPLICATION TO EXISTENCE OF THREE SOLUTIONS FOR A DIRICHLET PROBLEM IN ONE-DIMENSIONAL CASE
}

\author{
G.A. AFROUZI*, S. HEIDARKHANI, H. HOSSIENZADEH, AND A. YAZDANI
}

ABstract. In this paper, we establish an equivalent statement of minimax inequality for a special class of functionals. As an application, a result for the existence of three solutions to the Dirichlet problem

$$
\left\{\begin{array}{l}
-\left(\left|u^{\prime}\right|^{p-2} u^{\prime}\right)^{\prime}=\lambda f(x, u), \\
u(a)=u(b)=0
\end{array}\right.
$$

where $f:[a, b] \times R \rightarrow R$ is a continuous function, $p>1$ and $\lambda>0$, is emphasized.

\section{INTRODUCTION AND PRELIMINARIES}

Given two Gâteaux differentiable functionals $\Phi$ and $T$ on a real Banach space $\mathrm{X}$, the minimax inequality

$$
\sup _{\lambda \geq 0} \inf _{u \in X}(\Phi(u)+\lambda(\rho-T(u)))<\inf _{u \in X} \sup _{\lambda \geq 0}(\Phi(u)+\lambda(\rho-T(u))), \rho \in R,
$$

plays a fundamental role for establishing the existence of at least three critical points for the functional $\Phi(u)-\lambda T(u)$.

In this paper some conditions that imply the minimax inequality (1) are point out and equivalent formulations are proved.

The main result of this paper (Theorem 2.1) establishes an equivalent statement of minimax inequality (1) for a special class of functionals, while its consequences

Date: Received: 2 March 2008; Revised: 02 Jan. 2010

* Corresponding author.

2000 Mathematics Subject Classification. 5J20; 34A15.

Key words and phrases. Minimax inequality; critical point; three solutions; multiplicity results; dirichlet problem. 
(Theorem 2.4 and Theorem 2.6) guarantee some conditions so that minimax inequality holds.

Finally, we apply Theorem $A$ to elliptic equations, by using an immediate consequence of Theorem 2.1, and we consider the boundary value problem

$$
\left\{\begin{array}{l}
-\left(\left|u^{\prime}\right|^{p-2} u^{\prime}\right)^{\prime}=\lambda f(x, u), \\
u(a)=u(b)=0
\end{array}\right.
$$

where $f:[a, b] \times R \rightarrow R$ is a continuous function, $p>1$ and $\lambda>0$, and we establish some conditions on $f$ so that problem (2) admits at least three weak solutions. We say that $u$ is a weak solution to $(2)$ if $u \in W_{0}^{1, p}([a, b])$ and

$$
\int_{a}^{b}\left|u^{\prime}(x)\right|^{p-2} u^{\prime}(x) v^{\prime}(x) d x-\lambda \int_{a}^{b} f(x, u(x)) v(x) d x=0
$$

for every $v \in W_{0}^{1, p}([a, b])$.

Also by a similar arguments as in the problem (2), we will have the existence of at least three weak solutions for the problem

$$
\left\{\begin{array}{l}
-\left(\left|u^{\prime}\right|^{p-2} u^{\prime}\right)^{\prime}=\lambda h_{1}(x) h_{2}(u), \\
u(a)=u(b)=0
\end{array}\right.
$$

where $h_{1} \in C([a, b])$ is a function and $h_{2} \in C(R)$ is a positive function, and for the problem

$$
\left\{\begin{array}{l}
-\left(\left|u^{\prime}\right|^{p-2} u^{\prime}\right)^{\prime}=\lambda f(u) \\
u(a)=u(b)=0
\end{array}\right.
$$

where $f: R \rightarrow R$ is a continuous function.

In recent years, many authors have studied multiple solutions from several points of view and with different approaches and we refer to [1-3] and the references therein for more details, for instance, in their interesting paper [1], the authors studied problem

$$
\left\{\begin{array}{l}
u^{\prime \prime}+\lambda f(u)=0 \\
u(0)=u(1)=0
\end{array}\right.
$$

(independent of $\lambda$, in the case) by using a multiple fixed-point theorem to obtain three symmetric positive solutions under growth conditions on $f$.

In particular, in [2], the author proves multiplicity results for the problem (5) which for each $\lambda \in\left[0,+\infty\left[\right.\right.$, admits at least three solutions in $W_{0}^{1,2}([0,1])$ when $f$ is a continuous function.

\section{MAIn RESUlts}

First, we recall the three critical points theorem of B. Ricceri [4] by choos$\operatorname{ing} h(\lambda)=\lambda \rho$ :

Theorem A. Let X be a separable and reflexive real Banach space; $\Phi: X \longrightarrow R$ a continuously Gâteaux differentiable and sequentially weakly lower semicontinuous functional whose Gâteaux derivative admits a continuous inverse on $X^{*}$; $\Psi: X \longrightarrow R$ a continuously Gâteaux differentiable functional whose Gâteaux 
derivative is compact.

Assume that

$$
\lim _{\|u\| \rightarrow+\infty}(\Phi(u)+\lambda \Psi(u))=+\infty
$$

for all $\lambda \in[0,+\infty[$, and that there exists $\rho \in R$ such that

$$
\sup _{\lambda \geq 0} \inf _{u \in X}(\Phi(u)+\lambda \Psi(u)+\lambda \rho)<\inf _{u \in X} \sup _{\lambda \geq 0}(\Phi(u)+\lambda \Psi(u)+\lambda \rho) .
$$

Then, there exists an open interval $\Lambda \subseteq[0,+\infty[$ and a positive real number $q$ such that, for each $\lambda \in \Lambda$, the equation

$$
\Phi^{\prime}(u)+\lambda \Psi^{\prime}(u)=0
$$

has at least three solutions in $\mathrm{X}$ whose norms are less that than $q$.

Here and in the sequel, $\mathrm{X}$ will denote the Sobolev space $W_{0}^{1, p}([a, b])$ with the norm

$$
\|u\|:=\left(\int_{a}^{b}\left|u^{\prime}(x)\right|^{p} d x\right)^{1 / p}
$$

$p>1, f:[a, b] \times R \rightarrow R$ is a continuous function and $g:[a, b] \times R \rightarrow R$ is the function defined as follows

$$
g(x, t)=\int_{0}^{t} f(x, \xi) d \xi
$$

for each $(x, t) \in[a, b] \times R$.

We now introduce two positive special functionals on the Sobolev space $\mathrm{X}$ as follows

for every $u \in X$, and

$$
\Phi(u):=\frac{\|u\|^{p}}{p}
$$

for every $u \in X$.

Let $\rho, r \in R, w \in X$ be such that $0<\rho<\Psi(w)$ and $0<r<\Phi(w)$. We put

$$
\begin{aligned}
& \beta_{1}(\rho, w):=\rho \frac{\Phi(w)}{T(w)}, \\
& \beta_{2}(r, w):=r \frac{T(w)}{\Phi(w)}
\end{aligned}
$$

and

$$
\beta_{3}(\rho, w):=\frac{1}{2}(b-a)^{\frac{p-1}{p}}\left(p \beta_{1}(\rho, w)\right)^{1 / p},
$$

Clearly, $\beta_{1}(\rho, w), \beta_{2}(r, w)$ and $\beta_{3}(\rho, w)$ are positive. Now, we put

$$
\begin{gathered}
\delta_{1}:=\inf \left\{\frac{1}{2}(b-a)^{\frac{p-1}{p}}\|u\| \in R^{+} ; T(u) \geq \rho\right\}, \\
\delta_{2}:=\inf \left\{\frac{1}{2}(b-a)^{\frac{p-1}{p}}\|u\| \in R^{+} ;(b-a) \max _{(x, t) \in[a, b] \times\left[-\frac{1}{2}(b-a)^{\frac{p-1}{p}}\|u\|, \frac{1}{2}(b-a)^{\frac{p-1}{p}}\|u\|\right]} g(x, t) \geq \rho\right\}
\end{gathered}
$$


and

$$
\delta_{\rho}:=\delta_{1}-\delta_{2}
$$

Clearly, $\delta_{1} \geq \delta_{2}$.

Taking into account that for every $u \in X$, one has

$$
|u(x)| \leq \frac{1}{2}(b-a)^{\frac{p-1}{p}}\|u\|
$$

for all $u \in X$ and for all $x \in[a, b]$, so that

$$
T(u)=\int_{a}^{b} g(x, u(x)) d x \leq(b-a) \max g(x, t)
$$

where $(x, t) \in[a, b] \times\left[-\frac{1}{2}(b-a)^{\frac{p-1}{p}}\|u\|, \frac{1}{2}(b-a)^{\frac{p-1}{p}}\|u\|\right]$.

Namely

$$
T(u) \leq(b-a) \max g(x, t),
$$

where $(x, t) \in[a, b] \times\left[-\frac{1}{2}(b-a)^{\frac{p-1}{p}}\|u\|, \frac{1}{2}(b-a)^{\frac{p-1}{p}}\|u\|\right]$;

therefore, the set $\left\{\frac{1}{2}(b-a)^{\frac{p-1}{p}}\|u\| \in R^{+} ; T(u) \geq \rho\right\}$ is a subset of the set $\left\{\frac{1}{2}(b-a)^{\frac{p-1}{p}}\|u\| \in R^{+} ;(b-a) \max _{(x, t) \in[a, b] \times\left[-\frac{1}{2}(b-a)^{\frac{p-1}{p}}\|u\|, \frac{1}{2}(b-a)^{\frac{p-1}{p}}\|u\|\right]} g(x, t) \geq \rho\right\}$.

So, we have

$$
\begin{aligned}
& \inf \left\{\frac{1}{2}(b-a)^{\frac{p-1}{p}}\|u\| \in R^{+} ; T(u) \geq \rho\right\} \geq \\
& \inf \left\{\frac{1}{2}(b-a)^{\frac{p-1}{p}}\|u\| \in R^{+} ;(b-a) \max _{(x, t) \in[a, b] \times\left[-\frac{1}{2}(b-a)^{\frac{p-1}{p}}\|u\|, \frac{1}{2}(b-a)^{\frac{p-1}{p}}\|u\|\right]} g(x, t) \geq \rho\right\} .
\end{aligned}
$$

Hence $\delta_{\rho} \geq 0$.

The main result of this paper is the following theorem:

Theorem 2.1. Assume that there exist $\rho \in R, w \in X$ such that

(i) $0<\rho<T(w)$,

(ii) $(b-a) \max _{(x, t) \in[a, b] \times\left[-\beta_{3}(\rho, w)+\delta_{\rho}, \beta_{3}(\rho, w)-\delta_{\rho}\right]} g(x, t)<\rho$;

where $\beta_{3}(\rho, w)$ is given by (8) and $\delta_{\rho}$ by $(9)$.

Then, there exists $\rho \in R$ such that

$$
\sup _{\lambda \geq 0} \inf _{u \in X}(\Phi(u)+\lambda(\rho-T(u)))<\inf _{u \in X} \sup _{\lambda \geq 0}(\Phi(u)+\lambda(\rho-T(u))) .
$$

Proof: From $(i i)$, we obtain

$$
\beta_{3}(\rho, w)-\delta_{\rho} \notin\left\{l \in R^{+} ;(b-a) \max _{(x, t) \in[a, b] \times[-l, l]} g(x, t) \geq \rho\right\} .
$$


Moreover

$$
\inf \left\{l \in R^{+} ;(b-a) \max _{(x, t) \in[a, b] \times[-l, l]} g(x, t) \geq \rho\right\} \geq \beta_{3}(\rho, w)-\delta_{\rho} ;
$$

in fact, arguing by contradiction, we assume that there is $l_{1} \in R^{+}$such that

$$
(b-a) \max _{(x, t) \in[a, b] \times\left[-l_{1}, l_{1}\right]} g(x, t) \geq \rho
$$

and

$$
l_{1}<\beta_{3}(\rho, w)-\delta_{\rho}
$$

SO

$$
(b-a) \max _{(x, t) \in[a, b] \times\left[-\beta_{3}(\rho, w)+\delta_{\rho}, \beta_{3}(\rho, w)-\delta_{\rho}\right]} g(x, t) \geq(b-a) \max _{(x, t) \in[a, b] \times\left[-l_{1}, l_{1}\right]} g(x, t) \geq \rho
$$

and this is a contradiction. So

$$
\inf \left\{l \in R^{+} ;(b-a) \max _{(x, t) \in[a, b] \times[-l, l]} g(x, t) \geq \rho\right\}>\beta_{3}(\rho, w)-\delta_{\rho} .
$$

Therefore,

$$
\begin{gathered}
\inf \left\{\frac{1}{2}(b-a)^{\frac{p-1}{p}}\|u\| \in R^{+} ;(b-a) \max _{(x, t) \in[a, b] \times\left[-\frac{1}{2}(b-a)^{\frac{p-1}{p}}\|u\|, \frac{1}{2}(b-a)^{\frac{p-1}{p}}\|u\|\right]} g(x, t) \geq \rho\right\} \\
>\beta_{3}(\rho, w)-\delta_{\rho} ;
\end{gathered}
$$

namely $\beta_{3}(\rho, w)<\delta_{1}$. So, we have

$$
\inf \left\{\frac{\|u\|^{p}}{p} \in R^{+} ; T(u) \geq \rho\right\}>\beta_{1}(\rho, w),
$$

namely

$$
\inf \left\{\Phi(u) ; u \in T^{-1}\left([\rho,+\infty[)\}>\rho \frac{\Phi(w)}{T(w)}\right.\right.
$$

and, taking in to account that $(i)$ holds, one has

$$
\frac{\inf \left\{\Phi(u) ; u \in T^{-1}([\rho,+\infty[)\}\right.}{\rho}>\frac{\Phi(w)-\inf \left\{\Phi(u) ; u \in T^{-1}([\rho,+\infty[)\}\right.}{T(w)-\rho} .
$$

Now, let $\lambda \in R$. Taking into account the previous inequality, one has either

or

$$
\lambda>\frac{\Phi(w)-\inf \left\{\Phi(u) ; u \in T^{-1}([\rho,+\infty[)\}\right.}{T(w)-\rho}
$$

Namely

$$
\lambda<\frac{\inf \left\{\Phi(u) ; u \in T^{-1}([\rho,+\infty[)\}\right.}{\rho} .
$$

$$
\inf \left\{\Phi(u) ; u \in T^{-1}([\rho,+\infty[)\}>\Phi(w)+\lambda(\rho-T(w))\right.
$$

or

$$
\lambda \rho<\inf \left\{\Phi(u) ; u \in T^{-1}([\rho,+\infty[)\} .\right.
$$

Therefore, thanks to the $0<\rho<T(w)$, we obtain

$$
\inf _{u \in X}(\Phi(u)+\lambda(\rho-T(u)))<\inf \left\{\Phi(u) ; u \in T^{-1}([\rho,+\infty[)\},\right.
$$


and then, one has

$$
\sup _{\lambda \geq 0} \inf _{u \in X}(\Phi(u)+\lambda(\rho-T(u)))<\inf \left\{\Phi(u) ; u \in T^{-1}([\rho,+\infty[)\} .\right.
$$

Therefore, thanks to the

$$
\inf _{u \in X} \sup _{\lambda \geq 0}(\Phi(u)+\lambda(\rho-T(u)))=\inf \left\{\Phi(u) ; u \in T^{-1}([\rho,+\infty[)\},\right.
$$

we have the

$$
\sup _{\lambda \geq 0} \inf _{u \in X}(\Phi(u)+\lambda(\rho-T(u)))<\inf _{u \in X} \sup _{\lambda \geq 0}(\Phi(u)+\lambda(\rho-T(u))) .
$$

Remark 2.2. $\sup _{\lambda \geq 0} \inf _{u \in X}(\Phi(u)+\lambda(\rho-T(u)))$ is well define, because $\lambda \rightarrow$ $\inf _{u \in X}(\Phi(u)+\lambda(\rho-\bar{T}(u)))$ is upper semicontinuous in $[0,+\infty[$ and tends to $-\infty$ as $\lambda \rightarrow+\infty$.

Remark 2.3. If in Theorem 2.1, $\beta_{3}(\rho, w)-\delta_{\rho} \leq 0$; the Theorem holds again. Because, $\beta_{3}(\rho, w) \leq \delta_{1}-\delta_{2} \leq \delta_{1}$. Arguing as before, proof Theorem 2.1, result holds.

If instead of condition (ii) in Theorem 2.1, we put

$$
(b-a) \max _{(x, t) \in[a, b] \times\left[-\beta_{3}(\rho, w), \beta_{3}(\rho, w)\right]} g(x, t)<\rho,
$$

then the result holds, because

$(b-a) \max _{(x, t) \in[a, b] \times\left[-\beta_{3}(\rho, w)+\delta_{\rho}, \beta_{3}(\rho, w)-\delta_{\rho}\right]} g(x, t) \leq(b-a) \max _{(x, t) \in[a, b] \times\left[-\beta_{3}(\rho, w), \beta_{3}(\rho, w)\right]} g(x, t)<\rho$.

So, we have the following result:

Theorem 2.4. Assume that there exist $\rho \in R, w \in X$ such that

(i) $0<\rho<T(w)$

(ii) $(b-a) \max _{(x, t) \in[a, b] \times\left[-\beta_{3}(\rho, w), \beta_{3}(\rho, w)\right]} g(x, t)<\rho$.

where $\beta_{3}(\rho, w)$ is given by $(8)$.

Then, there exists $\rho \in R$ such that

$$
\sup _{\lambda \geq 0} \inf _{u \in X}(\Phi(u)+\lambda(\rho-T(u)))<\inf _{u \in X} \sup _{\lambda \geq 0}(\Phi(u)+\lambda(\rho-T(u))) .
$$

Now, we point out the following result:

Proposition 2.5. The following assertions are equivalent:

(a) there are $\rho \in R, w \in X$ such that

(i) $0<\rho<T(w)$

(ii) $(b-a) \max _{(x, t) \in[a, b] \times\left[-\beta_{3}(\rho, w), \beta_{3}(\rho, w)\right]} g(x, t)<\rho$;

where $\beta_{3}(\rho, w)$ is given by (8). 
(b) there are $r \in R, w \in X$ such that

(j) $0<r<\Phi(w)$

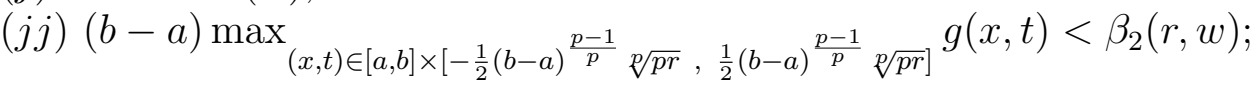

where $\beta_{2}(r, w)$ is given by $(7)$.

\section{Proof:}

$(a) \Rightarrow(b)$. First we note that $0<\Phi(w)$, because if $0 \geq \Phi(w)$, from $(i)$ one has $\rho \frac{\Phi(w)}{T(w)} \geq \Phi(w)$, namely $\beta_{3}(\rho, w) \geq \frac{1}{2}(b-a)^{\frac{p-1}{p}}\|w\|$. Hence, taking into account (ii), one has

$$
\begin{aligned}
T(w) & \leq(b-a) \max _{(x, t) \in[a, b] \times\left[-\frac{1}{2}(b-a)^{\frac{p-1}{p}}\|w\|, \frac{1}{2}(b-a)^{\frac{p-1}{p}}\|w\|\right]} g(x, t) \\
& \leq(b-a) \max _{(x, t) \in[a, b] \times\left[-\beta_{3}(\rho, w), \beta_{3}(\rho, w)\right]} g(x, t) \\
& <\rho,
\end{aligned}
$$

and that is in contradiction to $(i)$. We now put $\beta_{1}(\rho, w)=r$. We obtain $\rho=$ $\beta_{2}(r, w)$ and $\beta_{3}(\rho, w)=\frac{1}{2}(b-a)^{\frac{p-1}{p}} \sqrt[p]{p r}$. Therefore, from $(i)$ and $(i i)$, one has

$$
0<r<\Phi(w)
$$

and

$$
(b-a) \max _{(x, t) \in[a, b] \times\left[-\frac{1}{2}(b-a)^{\frac{p-1}{p}} \sqrt[p]{p r}, \frac{1}{2}(b-a)^{\frac{p-1}{p}} \sqrt[p]{p r}\right]} g(x, t)<\beta_{2}(r, w) .
$$

$(b) \Rightarrow(a)$. First we note that $0<T(w)$, because if $0 \geq T(w)$, from $(j)$ one has $r \frac{T(w)}{\Phi(w)} \leq 0$; namely, $\beta_{2}(r, w) \leq 0$. Hence, from $(j j)$ one has

$$
0=T(0) \leq(b-a) \max _{(x, t) \in[a, b] \times\left[-\frac{1}{2}(b-a)^{\frac{p-1}{p}} \sqrt[p]{p r}, \frac{1}{2}(b-a)^{\frac{p-1}{p}} \sqrt[p]{p r}\right]} g(x, t)<0,
$$

and this is a contradiction. We now put $\beta_{2}(r, w)=\rho$. We obtain $r=\beta_{1}(\rho, w)$ and $\frac{1}{2}(b-a)^{\frac{p-1}{p}} \sqrt[p]{p r}=\beta_{3}(\rho, w)$. Therefore, from $(j)$ and $(j j)$, we have the conclusion.

The following Theorem is another consequence of Theorem 2.1.

Theorem 2.6. Assume that there exist $r \in R, w \in X$ such that

(j) $0<r<\Phi(w)$ $(j j)(b-a) \max _{(x, t) \in[a, b] \times\left[-\frac{1}{2}(b-a)^{\frac{p-1}{p}}\right.} \sqrt[p]{p r}, \frac{1}{2}(b-a)^{\frac{p-1}{p}} \sqrt[p]{p r]} g(x, t)<\beta_{2}(r, w)$

where $\beta_{2}(r, w)$ is given by $(7)$.

Then, there exists $\rho \in R$ such that

$$
\sup _{\lambda \geq 0} \inf _{u \in X}(\Phi(u)+\lambda(\rho-T(u)))<\inf _{u \in X} \sup _{\lambda \geq 0}(\Phi(u)+\lambda(\rho-T(u))) .
$$


Proof: It follows from Theorem 2.4 and Proposition 2.5.

Finally, we interested in ensuring the existence of at least three weak solutions for the Dirichlet problem (2) where $f:[a, b] \times R \rightarrow R$ is a continuous function.

Now, we have the following result:

Theorem 2.7. Assume that there exist $\rho \in R, a_{1} \in L^{1}([a, b]), w \in X$ and a positive constant $\gamma$ with $\gamma<p$ such that

(i) $0<\rho<\int_{a}^{b} g(x, w(x)) d x$,

(ii) $(b-a) \max _{(x, t) \in[a, b] \times\left[-\beta_{3}(\rho, w), \beta_{3}(\rho, w)\right]} g(x, t)<\rho$.

(iii) $g(x, t) \leq a_{1}(x)\left(1+|t|^{\gamma}\right)$ almost everywhere in $[a, b]$ and for each $t \in R$.

where $\beta_{3}(\rho, w)$ is given by (8).

Then, there exists an open interval $\Lambda \subseteq[0,+\infty$ [ and a positive real number $q$ such that, for each $\lambda \in \Lambda$, problem (2) admits at least three solutions in X whose norms are less than $q$.

Proof: For each $u \in \mathrm{X}$, we put

$$
\begin{gathered}
\Phi(u)=\frac{\|u\|^{p}}{p}, \\
\Psi(u)=-\int_{a}^{b} g(x, u(x)) d x
\end{gathered}
$$

and

$$
J(u)=\Phi(u)+\lambda \Psi(u)
$$

In particular, for each $u, v \in X$ one has

$$
\Phi^{\prime}(u)(v)=\int_{a}^{b}\left|u^{\prime}(x)\right|^{p-2} u^{\prime}(x) v^{\prime}(x) d x
$$

and

$$
\Psi^{\prime}(u)(v)=-\int_{a}^{b} f(x, u(x)) v(x) d x .
$$

It is well known that the critical points of $J$ are the weak solutions of (2), our goal is to prove that $\Phi$ and $\Psi$ satisfy the assumptions of Theorem A. Clearly, $\Phi$ is a continuously Gâteaux differentiable and sequentially weakly lower semi continuous functional whose Gâteaux derivative admits a continuous inverse on $X^{*}$ and $\Psi$ is a continuously Gâteaux differentiable functional whose Gâteaux derivative is compact.

Thanks to (iii), for each $\lambda>0$ one has that

$$
\lim _{\|u\| \rightarrow+\infty}(\Phi(u)+\lambda \Psi(u))=+\infty
$$


for all $\lambda \in[0,+\infty[$.

Furthermore, thanks to Theorem 2.4, from $(i)$ and $(i i)$ we have

$$
\sup _{\lambda \geq 0} \inf _{u \in X}(\Phi(u)+\lambda \Psi(u)+\lambda \rho)<\inf _{u \in X} \sup _{\lambda \geq 0}(\Phi(u)+\lambda \Psi(u)+\lambda \rho) .
$$

Therefore, we can apply Theorem $A$. It follows that there exists an open interval $\Lambda \subseteq[0,+\infty$ [ and a positive real number $q$ such that, for each $\lambda \in \Lambda$, problem (2) admits at least three solutions in X whose norms are less than $q$.

We also have the following existence result:

Theorem 2.8. Assume that there exist $r \in R, a_{2} \in L^{1}([a, b]), w \in X$ and a positive constant $\gamma$ with $\gamma<p$ such that

(j) $0<r<\frac{\|w\|^{p}}{p}$

$(j j)(b-a) \max _{(x, t) \in[a, b] \times\left[-\frac{1}{2}(b-a)^{\frac{p-1}{p}} \sqrt[p]{p r}, \frac{1}{2}(b-a)^{\frac{p-1}{p}} \sqrt[p]{p r]}\right.} g(x, t)<\beta_{2}(r, w)$;

$(j j j) g(x, t) \leq a_{2}(x)\left(1+|t|^{\gamma}\right)$ almost everywhere in $[a, b]$ and for each $t \in R$.

where $\beta_{2}(r, w)$ is given by $(7)$.

Then, there exists an open interval $\Lambda \subseteq[0,+\infty$ [ and a positive real number $\mathrm{q}$ such that, for each $\lambda \in \Lambda$, problem (2) admits at least three solutions in $\mathrm{X}$ whose norms are less than q.

Proof: It follows from Theorem 2.6 and Theorem 2.7.

Let $h_{1} \in C([a, b])$ be a function and $h_{2} \in C(R)$ be a positive function. Put

$$
f(x, t)=h_{1}(x) h_{2}(t)
$$

for each $(x, t) \in[a, b] \times R$,

$$
\alpha(t)=\int_{0}^{t} h_{2}(\xi) d \xi
$$

for all $t \in R$, and

$$
a_{3}(x)=\frac{a_{1}(x)}{h_{1}(x)}
$$

for almost every $x \in[a, b]$. Then, with use the Theorem 2.7 , we have the following result:

Theorem 2.9. Assume that there exist $\rho \in R, a_{3} \in L^{1}([a, b]), w \in X$ and a positive constant $\gamma$ with $\gamma<p$ such that

$\left(i^{\prime}\right) 0<\rho<\int_{a}^{b}\left(h_{1}(x) \alpha(w(x))\right) d x$

$\left(i i^{\prime}\right)(b-a) \max _{x \in[a, b]} h_{1}(x)<\frac{\rho}{\alpha\left(\beta_{3}(\rho, w)\right)}$.

$\left(i i i^{\prime}\right) \alpha(t) \leq a_{3}(x)\left(1+|t|^{\gamma}\right)$ almost everywhere in $[a, b]$ and for each $t \in R$.

where $\beta_{3}(\rho, w)$ is given by $(8)$. 
Then, there exists an open interval $\Lambda \subseteq[0,+\infty[$ and a positive real number $q$ such that, for each $\lambda \in \Lambda$, problem (3) admits at least three solutions in $\mathrm{X}$ whose norms are less than $q$.

Put

$$
a_{4}(x)=\frac{a_{2}(x)}{h_{1}(x)}
$$

for almost every $x \in[a, b]$. Then, with use the Theorem 2.8, we have the following existence result:

Theorem 2.10. Assume that there exist $r \in R, a_{4} \in L^{1}([a, b]), w \in X$ and a positive constant $\gamma$ with $\gamma<p$ such that

$\left(j^{\prime}\right) 0<r<\frac{\|w\|^{p}}{p}$

$\left(j j^{\prime}\right)(b-a) \max _{x \in[a, b]} h_{1}(x)<\frac{\beta_{2}(r, w)}{\alpha(\theta)}$;

$\left(j j j^{\prime}\right) \alpha(t) \leq a_{4}(x)\left(1+|t|^{\gamma}\right)$ almost everywhere in $[a, b]$ and for each $t \in R$.

where $\theta=\frac{1}{2}(b-a)^{\frac{p-1}{p}} \sqrt[p]{p r}$ and $\beta_{2}(r, w)$ is given by (7).

Then, there exists an open interval $\Lambda \subseteq[0,+\infty$ [ and a positive real number $q$ such that, for each $\lambda \in \Lambda$, problem (3) admits at least three solutions in X whose norms are less than $q$.

We now want to point out two simple consequences of Theorem 2.7 and Theorem 2.8, respectively. Let $f: R \rightarrow R$ be a continuous function. Put $g(t)=$ $\int_{0}^{t} f(\xi) d \xi$ for each $t \in R$.

So we have the following results:

Theorem 2.11. Assume that there exist $\rho \in R, w \in X$ and two positive constants $\gamma$ and $\eta$ with $\gamma<p$ such that

$\left(i^{\prime \prime}\right) 0<\rho<\int_{a}^{b} g(w(x)) d x$,

$\left(i i^{\prime \prime}\right)(b-a) \max _{t \in\left[-\beta_{3}(\rho, w), \beta_{3}(\rho, w)\right]} g(t)<\rho$.

$\left(i i i^{\prime \prime}\right) g(t) \leq \eta\left(1+|t|^{\gamma}\right)$ for each $t \in R$.

where $\beta_{3}(\rho, w)$ is given by (8).

Then, there exists an open interval $\Lambda \subseteq[0,+\infty[$ and a positive real number $q$ such that, for each $\lambda \in \Lambda$, problem (4) admits at least three solutions in X whose norms are less than $q$.

Theorem 2.12. Assume that there exist $r \in R, w \in X$ and two positive constants $\gamma$ and $\mu$ with $\gamma<p$ such that

$$
\begin{aligned}
& \left(j^{\prime \prime}\right) 0<r<\frac{\|w\|^{p}}{p}, \\
& \left(j j^{\prime \prime}\right)(b-a) \max _{t \in\left[-\frac{1}{2}(b-a)^{\frac{p-1}{p}} \sqrt[p]{p r}, \frac{1}{2}(b-a)^{\frac{p-1}{p}} \sqrt[p]{p r}\right]} g(t)<\beta_{2}(r, w) ;
\end{aligned}
$$


$\left(j j j^{\prime \prime}\right) g(t) \leq \mu\left(1+|t|^{\gamma}\right)$ for each $t \in R$.

where $\beta_{2}(r, w)$ is given by $(7)$.

Then, there exists an open interval $\Lambda \subseteq[0,+\infty$ [ and a positive real number $q$ such that, for each $\lambda \in \Lambda$, problem (4) admits at least three solutions in X whose norms are less than $q$.

\section{REFERENCES}

[1] R.I. Avery, J. Henderson, Three symmetric positive solutions for a second-order boundary value problem, Appl. Math. Lett. 13 (2000) 1-7.

[2] G. Bonanno, Existence of three solutions for a two point boundary value problem, Appl. Math. Lett. 13 (2000) 53-57.

[3] P. Korman, T. Ouyang, Exact multiplicity results for two classes of boundary value problem, Diff. Integral Equations 6 (1993) 1507-1517.

[4] B. Ricceri, On a three critical points theorem, Arch. Math. (Basel) 75 (2000) 220-226.

Department of Mathematics, Faculty of Basic Sciences, University of MazanDARAN, BABOLSAR, IRAN

E-mail address: afrouzi@umz.ac.ir 